\title{
ARGUING ABOUT PLANNING ALTERNATIVES
}

\author{
ALEX QUILICI \\ Department of Electrical Engineering \\ 2540 Dole Street, Holmes Hall 455 \\ University of Hawaii at Manoa \\ Honolulu, HI, 96822
}

\begin{abstract}
In discourse processing, two major problems are understanding the underlying connections between successive dialog utterances and deciding on the content of a coherent dialog response. This paper presents a computational model of these tasks for a restricted clase of argumentative dialogs. In these dialogs, each response presents a belief that justifies or contradicts another belief presented or inferred earlier in the dialog. Understanding a response involves relating a stated belief to these earlier beliefs, and producing a response involves selecting a belief to justify and deciding upon the set of beliefs to provide as its justification. Our approach is knowledge based, using general, common-sense justification rules to recognire how a belief is being justified and to form new justifications for beliefs. This approach provides the ability to recognise and respond to never before seen belief juatifications, a necessary capability for any system that participates in dialogs involving disagreements.
\end{abstract}

\section{Introduction}

In discourse processing, two major problems are underatanding the underlying connections between succeasive dialog reaponses and deciding on the content of a coherent dialog response. This paper presents an initial model that accomplishes these tasks for one class of argumentative dialogs. In this class, each dialog response presents a belief that justifies or contradicts a belief provided earlier in the dialog.

The following dialog fragment is an example:

(1) TrDY: The members of the AI lab should clean it themselves.

(2) SCRUPrY: But that interferes with doing research.

(3) TiDy: There's no other way to keep it clean.

(4) Scrufpy: We can pay a janitor to keep it clean.

(5) TidY: We need money to pay a janitor.

(6) Scrupry: We can transfer the money from the salary fund.

(7) TIDY: But doing that interferes with paying the lab members.
(8) ScRUPFY: It's more desirable to have a clean lab than to pay the lab members.

Each response states one or more plan-oriented beliefs, usually as part of a whort chain of reasoning justifying or contradicting a belief provided earlier in the dialog.

In (1), TIDY begins by stating a belief: the lab members should execute the plan of cleaning the lab.

In (2), Scrupry responds with a belief that the lab members executing this plan interferes with their doing research. This belief justifies Scrurry's unstated belief that the lab members should not execute the plan of cleaning the lab, which contradicts TIDY's stated belief in (1). Scrupry's underlying reasoning is that the lab members shouldn't clean the lab because it interferes with their executing the more desirable plan of doing research.

In (3), Tiny presents a belief that there's no alternative plan for keeping the lab clean. This belief justifies TIDY's belief in (1). TIDY's underlying reawoning is that the lab members should clean the lab because it's the best plan for the goal of keeping the lab clean, and it's the best plan because it's the only plan that achieves the goal.

Finally, in (4), Scrurry states a belief that paying a janitor achieves the goal of keeping the lab clean. This contradicts TiDy's atated belief in (3). It also justifies a belief that the lab members cleaning the lab isn't the best plan for keeping the lab clean, which contradicts one of the beliefs inferred from (3). Scrufry's reasoning is that paying a janitor is a more desirable plan that achieves this goal.

The remaining responses follow the tame pattern. Understanding responses like these involves relating a stated belief to beliefs appearing earlier in the dialog. That requires inferring the participant's underlying reasoning chain and the beliefs it juntifies. Producing these responses involves selecting a belief to justify and deciding upon the set of beliefs to provide as its justification. That requirea constructing an appropriate reasoning chain that justifies holding any unshared beliefs.

Our focus in this paper is on an initial method for representing, recognising, and producing the belief justifications underlying dialog responses that provide coherent defenses of why beliefs are held. 
The behevior modeled is limited in several signifcant waye. First, we do not try to recognise when an arguer's respone contradicts one of his earlier responses, such as the contradiction between (2) and (8), nor do we try to avoid producing such responses. Second, we do not try to recognise or make une of high-level arguing atrategies, wuch es reductio ad absurdum. Third, we restrict ourselves to a $\mathrm{mmall}$ claes of belieft involving planning. Finally, we atart with representstions of beliefs and ignore the linguietic issues involved in turning responses into beliefs. Clearly, all these limitations must eventually be addreased in order to produce a more realistic model of debate. Our belief, however, is that an initial model of the process of recognising and producing belief juatifications is a ureful and necessary first step.

\section{Our Approach}

Our approach to these task rests on a simple asumption: Dialog participants justify beliefs with instantiations of general, common-sense justification rules. For plan-oriented beliefs, a justification rule correspond to a planning heuristic that's based solely on structural features of plans in general, not on characteristics of specific plans themselves.

The first few responses in this dialog illustrate several justification rules. In (2), Scrupry uses the rule: One reason why a plan shouldn't be executed is that it conflicts with executing a more desirable plan. Similarly, in (3), Tiny chains together a pair of these rules: One reason why a plan should be executed is that it's the best plan for achieving a goal, and One reason why a plan is the best plan for a goal is that it's the only plan that achieves the goal.

Given our assumption, understanding a response is equivalent to recognising which justification rules were chained together and instantiated to form it, determining which belief to address in a response is equivalent to determining which beliefs in a chain of instantiated justification rules are not shared, and producing a justification is equivalent to selecting and instantiating justification rules with beliefs from memory.

We make this assurnption for two reasons. First, dialog participants should be able to understand and respond to never before seen belief justifications. That suggests applying general knowledge, auch as our justification rules, to analyse and produce specific justifications, as that knowledge is likely to be shared by different participants, even if they hold different beliefs sbout specific courses of action. And second, dialog participants should aleo be able to we the same knowledge for different tasks. That suggents that arguments about planning should use the same knowledge as planning itself. The justifica- tion rulew for plen-oriented beliefo dewcribe knowledge that a planner would also find useful in selecting or constructing new plans.

Our approach differs in two ways from previous model of participating in dialoga. Firat, these modele emphasised plan recognition: the task of recognising and inferring the underlying plane and goaln of a dialog participant $[4,10,17,18,2]$. They view utterances as providing ateps in plans (typically by describing goalu or actions) and tie them together by inferring an underlying plan. But in an argument not only must the participant's plans and goals be inferred, but also their underlying beliefa about those plans and goals. Our approach suggests a model that infers these beliefs as a natural convequence of trying to understand connections between succeasive dialog utterances. In contrast, exinting approaches to inferring participant beliefa take a stated belief and try to reason about possible justifications for it [12, 9]. Previous models have also tended to view providing a dialog response solely as a part of the question answering process. In contrast, our appronch nuggests that responses arise as a natural consequence of trying to integrate newly-encountered beliefs with current beliefs in memory, and trying to underatand any contradictions that result.

\section{Justification Rules}

The argumentative dialoge we've examined have two types of plan-oriented beliefs: factual and evaluative [1]. Factual beliefs are objective judgements about planning relationships, such as whether a plan has a particular effect or enablement. They represeat the planning knowledge held by most previous plan-understanding and plan-constructing systems. Evaluative beliefs, on the other hand, are subjective judgements sbout planning relationships, such as whether or not a plan should be executed. Although these beliefs have generally been ignored by previous systems, they are crucial to participating in arguments involving plan-oriented beliefs.

Our assumption is there exists a umall set of justification rules for each planning relationship. Each rule is represented as an abstract configuration of planning relationships that, when instantiated, provides a reason for holding a particular belief. For example, the rule that a plan shouldn't be executed if it conflicts with a preferred plan is represented as:

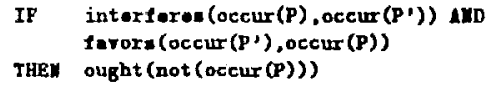

That is, a plan sbouldn't be executed if (1) it interferes with another plan, and (2) that plan is preferred to it. Figure 1 lists our current justification rules for 
Hexson why executing plsn $X$ is devirable:

$X$ is the beet plas los a gotl.

Executing $X$ is an exablement for a gool.

Reswom why executing plan $X$ is undesirable:

$\mathbf{X}$ conflicts with o tnore desirable plan.

$X$ bas an undesirable effext.

$X$ ba an undevirable enablement.

Heanons why plan $X$ is the best plan for a gol:

$X$ is the only plas that achieves the gonl.

No plan more deairable then $X$ achieves the goul.

Reason why plan $X$ is not the best plan for a goul:

$X$ has en unmchievable enablentent.

$X$ 's execution is undesirable.

Some more desirable plan actieve. the gou!

Henoons why plen $X$ is more desirable than plan $Y$ : $\mathrm{X}$ has a desirable effect that $Y$ doean't have. $X$ doean't have an undeairable effect that $Y$ hob.

$X$ doean't have an undeairable enablement that $Y$ has. $Y$ conficts with more devirable pian and $X$ doesn' $t$. $X$ is an enablement of a more desirable plan than $Y$.

$X$ has an effect more desirable than $Y$.

Heasons why achieving gosl $G$ is undesirable:

The only plan for achieving $G$ is undesirable.

Achieving $G$ has an undexiruble effect $S$.

Heasons why achieving goal $G$ is desirable:

Achieving $G$ is an enablement for another gool.

Not achieving $G$ has an undesirable eftect $S$.

Figure 1: Justification rules.

evaluative beliefu (bee [13] for representational detuils and criteria for deciding what is a reasonable justification rule). These rules were abstracted from examining a variety of different plan-oriented argumentative dialogi.

The power of these justification rules comes from their generality: A aingle rule can be instantiated in different ways to provide justifications for different beliefs. In (2), Scnupry uses the above rule to justify a belief that the lab members shouldn't clean the lab themselves. In (7), TIDY uses the same rule to justify a belief that the lab members shouldn't transfer money from the salary fund. Here, TIDY's justification is that transferring the money interferes with the more desirable plan of paying researchers.

\section{Recognizing Justifications}

The process of understanding a dialog response is snodeled as a forward-chaining search for a chain of instartiated justitication rules that (1) contains the user's atated belief, and ( $(2)$ justifies an earlier dialog belief or its negation.

We briefly illustrate thin process by showing how Scruypy understands Troy's response in (3). The input belief is that the lab members cleaning the lab is the only plan that achievea the goal of keeping the lab clean. This belief matches an antecedent in a pair of justification rules, so the process beginn by instantiating these rules, resulting in pair of pomable justification chain that contain TIDY's stated belief: (1) the lab members cleaning is the best plan for heep. ing the lab clean because it's the only plan for keeping the lab clean, and (2) the lab shouldn't be kept clean because the only plan for that goal is the andesirable plan of having the lab members cleaning it.

Neither justification directly relates to the dialog, so the next step is to determine which one to puraue further, and whether either can be eliminated from further consideration. Here, the second justification contains a belief that the lab members cleaning the lab is undesirable, which contradicts Troy's stated belief in (1). Applying the heuristic Discand any potential justification containing beliefs that contradict the speaker's earlier beliefs" leaves only the first justification to pursue further. It's consequent is the antecedent of a single justification rule, and instantiating this rule leads to this justification chain: the lab members should clean the lab because their cleaning the lab is the best plan for the goal of keeping the lab clean because it's the only plan for keeping the lab clean. The justified belief is Troy's belief in (1), so the process stops.

In general, the underatanding process is more complex, since justification rules may not be completely instantiated by a single antecedent, and may therefore need to be further instantiated from belief in the dialog context and memory. There also may be many possible chains to pursue even after heuristically discarding some of them, requiring the une of other heuristics to determine which path to follow, such as "Pursue the reasoning chain which contains the most beliefo found in the dialog context."

\section{Selecting A Belief To Justify}

After recognizing a participant's reasoning chain, it's necessary to select a belief to justify as a response. This task involves determining which beliefs are not shared, and selecting the negation of one of those beliefs to justify.

An intuitive notion of agreement is that a belief is shared if it it's found in memory or can be justified, and it's not shared if its negation is found in memory or can be justified. But this notion is computationally expensive, since it could conceivably involve trying to justify all the beliefs in the participant's reasoning chain, as well as their negations. As anl alternative, our model determines whether a belief is shared by searching memory for the belief and its negation and, if that fails, applying a amall 
wet of agreement heuristics. One auch heuriatic in "Aosume a belief is shared if a justifying generalization is found in memory". So, for example, if the belief "keep everything clean" is found in memory, the belief "keep the AI lab clean" is considered to be shured. If no agreement heuristic applies, the belief is imply marked an "unknown".

After determining whether each belief in the participant'n reseoning chain in shared, the model first mearches for an exinting juutification for an unshared belief'a negation. If that faila, it then tries to create a new juatification for an unshared belief's negation. And if that fails, it tries to create a new justification for the negation of one of the unknown beliefs. This way existing justifications are presented before an attempt is made to construct new ones. If none of these steps succeed, the assumption is that the reasoning chain is shared, and an attempt is made to form a new justification for the belief it contradicts.

Thus, the belief our model addresses in a response arises from trying to discover whether or not it agrees with another participant's reasoning.

\section{Forming Justifications}

To form a new justification for a belief, our model performs a backward chaining search foi a chain of justification rules that justify the given belief and that can be inatantiated with beliefs from memory.

We briefly illustrate this process by showing how ScRurpy form the reaponse in (2). The belief to justify is that it's not desirable to have the lab members clean the lab. The first step is to instantiate the justification rules that have this belief as their consequent. That results in several possible justifications: (1) there's an wndesirable enablement of cleaning the lab, (2) there's an undesirable effect of cleaning the lab, or (3) the lab members cleaning the lab conflicts with a more desirable action.

The next step is to try to fully instantiate one of these rules. Applying the heuristic "Pursue the most instantiated justification rule" suggests working on the last rule. Here, Scrupry instantiates it with a belief from mernory that research is more desirable than cleaning. Once a rule is instantiated, it's necessary to verify that the beliefs it contains are shared. Here, that involves verifying that cleaning conflicts with research. It does, so the instantiated rule can be presented as the response.

In general, the process is more complex than outlined bere, since not all of the belief in an instantiated justification rule may be shared, and there may be meveral wayn to instantiate a particular rule. Those rules containing unknown beliefs require further justification, while those rules containing unshared beliefs can be discarded.

\section{Background}

The closest related system is ABDUL/ILANA [s], which debated the responsibility and cause for historical events. It focused on the complementary protlem of recognising and providing episodic justifice tions, rather than juatificationu based on the relu. tionshipa between different plans.

There are weveral models for recognixing the re. lationship between argument proponitions. Cohen's [5] takes each sew belief and checks it for a juatification relationship with a subset of the previously stated beliefs determined through the une of di. alog structure and clue words. That model a sumes the existence of an evidence oracle capable of determining whether a justification relationship holds between any paix of beliefs. Our model im plements this oracle for a particular cluse of plan oriented belief justifications. OpFd [3] recognives bo lief justifications in editorials about economic planning through the use of ergument units, nnowl edge atructure that can be viewed as complex configurations of justificatiou rules. The approaches are complementary, just as scripts [7] and plans [6, 18] are both useful methods for recognising the connections between events in a narrative.

Several systems have concentrated on producing belief justifications. Our own earlier work [14, 15, 16] used a primitive form of justification rules for factual beliefs as a template for producing corretive responses for user misconceptions. Our current model extends this work to use these rules in both understanding and responding, and provides additional rules for evaluative beliefs.

ROMPER [11] provides justification for beliefs about an object'a class or attributes. But it provides these justifications purely by template matching, not by constructing more general reasoning chains.

\section{Current Status}

We've completely implemented the model discused in this paper. The program is written in Quintus Prolog and runs on an IIP/APOLLO workstation. Its input is a representation for a stated participant belief, and its output is as representation for an ap. propriate response. It currently includes 30 justifica tion rules and over 400 beliefs about various plens. We've used the program to participate in short argumentative dialogs in two disparate domains: day. to-day planning in the AI lab, and removing and recovering files in UNIX. We're currently uning it to experiment with different beuristice for controlling the search proceas involved in recognixing and con. structing these reasoning cheins.

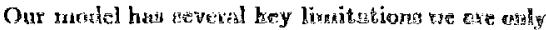


now starting to addrea. Pirnt, it viewe plan as atomic units and consider only a small set of "all or nothing" plan-oriented beliefs. This means it can't produce or understand juatifications involving stepa in a plan, conditioaal planning relationshipa, or belief not directly involving plank. Second, our model can underutand only those responses that juatify an earlier belief. It can't, for example, underntand a response that contradicts an inferred justification for an earlier belief. These more complex relationshipa can be represented uning justification rules, but our model muat be extended to recognise them. Third, our model is reactive rather than initiatory: it produces responses only when there's a perceived disagreement. It needs to be extended to know why its in an argument, and to be aware of the underlying goalu of the other argument participants.

\section{Conclusions}

Previous dialog models have focused primarily on recognising a participant's plans and goals. But to participate in an argument it's also necessary to recognise when participants are providing beliefs about their plana and goals and how they're justifying these beliefa. It's also necessary to be able to determine which beliefs require further justification and to formulate justifications for these beliefs. This paper suggeats a knowledge-based approach for these tasks.

Our approach has everal attractive features. Firat, it builda a model of many relevant but unstated participant beliefs as a side-effect of trying to relate their utterance to the dialog. Second, it decides which belief to address in a response as a natural consequence of trying to understand why it disagrees with another participant's belief. Third, it understands belief justifications uring the same general, common-sense planning knowledge that it uses to formulate them. Finally, it suggests how never before seen belief justifications can be understood, so long as they were formed from general justification rules known to the participants. That ability is crucial for participating in dialogs whose participants hold differing beliefs.

\section{References}

[1] R. Abelson. Differences Between Beliefs and Knowledge Systems. Cognitive Science 3, 1979.

[2] J.F. Allen and C.R. Perrault, Analyzing Intention in Utterances. Artificial Intelligence 15, 1980.

[3] S.J. Alvarado. Understanding Editorial Tezt: A Computer Model of Argument Comprehension. Kluwer, Boston, MA, 1990.
[4] S. Carberry. Modeling the Uner'a Plane and Goals. Computational Linguistics, 14(3), 1988.

(5) R. Cohen. Analyring the Structure of Argumentative Discourse. Computational Linguintics, $13(1), 1987$.

[6] M.G. Dyer. In-depth Underotanding: A Computer Model of Narrative Comprehension. MIT Press, Cambridge, MA, 1983.

[7] M.G. Dyer, R.E. Cullingford, and S.J. Alvarado. Scripts. In Encyclopedia of A rifieial Intelligence, John Wiley, NY, NY, 1987.

[8] M. Flowers, R. McGuire, and L. Birnbaum. Adversary Arguments and the Logic of Personal Attacks. In Strategies for Natural Language Proceseing. Lawrence Erlbaum, Hilladale, NJ, 1982.

[9] R. Kass. Building a User Model Implicitly from a Cooperative Advisory Dialog. User Modeling and User-Adapted Interaction, 1(3), 1991.

[10] D.J. Litman and J.F. Allen. A Plan Recognition Model for Subdialogues in Conversations. Cognitive Science, 11, 1987.

[11] K. McCoy. Reasoning on a Higblighted Uner Model to Respond to Misconceptions. Computetional Linguistics, 14(3), 1988.

[12] M. Pollack. Inferring Domain Plans in Question-Answering. $\mathrm{PhD}$ Thesis, Univernity of Pennsylvania, Philadelphia, PA, 1986.

[13] A. Quilici. The Correction Machine: A Computer Model Of Recognizing and Producing Belief Justifications in Argumentative Dialogs. PhD Thesis, University of California, LA, CA, 1991.

[14] A. Quilici. Participating In Plan-Oriented Dialogs. In Proceedings of the 12 th Conference of the Cognitive Science Society, Boston, MA, 1990.

[15] A. Quilici. The Correction Machine: Formulating Explanations for User Misconceptions. In Proceedings of the 1989 International Joint Conference on Artificial Intelligence, Detroit, MI, 1989.

[16] A. Quilici, M.G. Dyer, and M. Flowers. Recogaising and Responding to Plan-oriented Misconceptions. Computational Linguistics, 14(3), 1988.

[17] C. L. Sidner. Plan Paraing For Intended Response Recognition in Diecourse. Computational Intelligence, 1(1), 1985.

[18] R. Wilensky. Planning end Underslanding. Addison Wesley, Reading, MA, 1983. 